\title{
Kinematic Analysis of Continuum Robot Consisted of Driven Flexible Rods
}

\author{
Yingzhong Tian, ${ }^{1,2}$ Mingxuan Luan, ${ }^{1} \mathrm{Xu}$ Gao, ${ }^{1}$ Wenbin Wang, ${ }^{3}$ and Long $\mathrm{Li}^{1,2}$ \\ ${ }^{1}$ School of Electrical and Mechanical Engineering and Automation, Shanghai University, Shanghai 200072, China \\ ${ }^{2}$ Shanghai Key Laboratory of Intelligent Manufacturing and Robotics, Shanghai 200072, China \\ ${ }^{3}$ Mechanical and Electrical Engineering School, Shenzhen Polytechnic, Guangdong 518055, China \\ Correspondence should be addressed to Long Li; lil@shu.edu.cn
}

Received 22 March 2016; Revised 31 August 2016; Accepted 14 September 2016

Academic Editor: Paolo Maria Mariano

Copyright (C) 2016 Yingzhong Tian et al. This is an open access article distributed under the Creative Commons Attribution License, which permits unrestricted use, distribution, and reproduction in any medium, provided the original work is properly cited.

This paper presents the kinematic analysis of a continuum bionic robot with three flexible actuation rods. Since the motion of the end-effector is actuated by the deformation of the rods, the robot structure is with high elasticity and good compliance and the kinematic analysis of the robot requires special treatment. We propose a kinematic model based on the geometry with constant curvature. The analysis consists of two independent mappings: a general mapping for the kinematics of all robots and a specific mapping for this kind of robots. Both of those mappings are developed for the single section and for the multisections. We aim at providing a guide for kinematic analysis of the similar manipulators through this paper.

\section{Introduction}

Nowadays, people have already found the incredible ability of adaptation, excellent locomotion, and capability of being dexterous to move in complex environment presented by snakes [1], elephant's trunk [2], and octopus tentacles [3] from nature. These outstanding performances have inspired researchers to explore further in the fields of flexible bionic robots.

Robinson and Davies [4] proposed that the classification of robots can be divided into continuum robots, discrete robots, and serpentine robots. Continuum robots [5] can bend into different shapes because of the unique mechanical structure and the way of actuation in order to generate the desired movement path. This new type of robots possesses good ability of bending and dexterity; therefore we can apply these robots in narrow and unstructured environment.

Currently, researchers have become increasingly interested in the design and control of continuum robots. Sun invented a cable-driven continuum robot [6] for colonoscopy, which consists of a flexible backbone, several disks, and driven cables. This flexible robot has two bending joints, and each joint has two degrees of freedom. The end-effector can move in all directions of the workspace due to the actuated cables. Sun used a method of geometry analysis to develop the kinematic model of the cable-driven robot.

Walker and his team invented continuum robots such as Oct-Arm [7] and Air-Octor [8] with coupled tendons; Jones and Walker [9] designed a bionic elephant's trunk consisting of springs, damping device, and metal disks; Festo designed Bionic Tripod 2.0 and Bionic Tripod 3.0, and a bionic robot seems like elephant's trunk based on pneumatic bellows called Bionic Assistant [10]. Simaan [11] invented a kind of bionic robot which seems like snake with flexible backbone and cable-driven, with the diameter of $0.0042 \mathrm{~m}$, and this robot was proposed for throat surgery; Gravagne et al. designed a kind of continuum robot called Clemson tentacle manipulator [12].

Researchers developed different methods to establish the kinematic models of continuum robots with different structures. Jones and Walker $[7,9]$ used a modified homogenous transformation matrix in terms of Denavit-Hartenberg (DH) type approach to develop and analyze the kinematic model of the continuum robot. They also simulated the parameters of the continuum robot's trunk. This method gave the transformation of a section of the continuum robot between the 


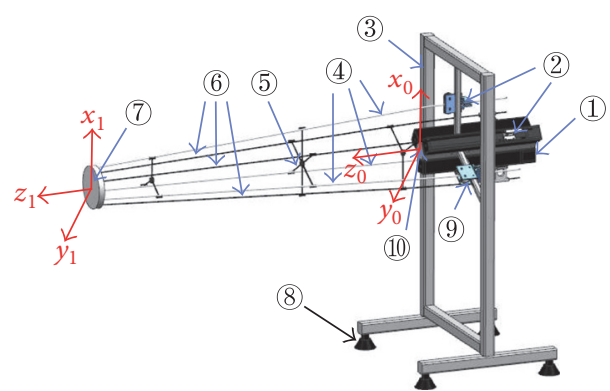

FIGURE 1: Prototype machine.

base coordinate frame and the coordinate frame of the endeffector by multiplying five $\mathrm{D}-\mathrm{H}$ transformation matrices. In the end, they obtained the homogenous transformation matrix of the robot. Their method reflected a transformation of robot kinematics model from rigid to flexible. Simaan et al. [13] used a differential method to divide the robot into several small units and then computed the position of the end-effector of a single manipulator by using integration method and obtained the Jacobian matrix.

In this paper, we design a bionic continuum robot inspired by fins, which uses flexible rods made of glass fiber to replace the rigid structure existing in the traditional robot. We can control the position and orientation of the endeffector by changing the length of the rods, and we use continuous curvature method based on geometry to develop kinematic model of the continuum robot. We obtain the mapping among the configuration space, manipulation space, and task space to analyze the kinematics.

\section{Design and Analysis of the Prototype Machine}

2.1. Design of the Prototype Machine. The prototype robot under study is shown in Figure 1, with its height of $1.2 \mathrm{~m}$ and its overall frame (3) made of aluminum alloy. The components of the prototype machine contain three modules (1), some parts of 3D printing (2), six fiber glass rods (4) and (6), three star configurations (5), four rubber base plates (8), twelve rollers (9), and any other fasteners. We assume that (10) is the base face.

The prototype robot has three passive rods (in gray as shown in Figure 1). The modules are fixed on the aluminum frame and distributed by 120 degrees. They are actuated by step motors. Three passive rods are auxiliary and installed through every four rollers, which are fixed on the corresponding aluminum alloy rods. Three active rods are covered by black like (6) as shown in Figure 1, with the length of $1.6 \mathrm{~m}$. Each active rod is fixed on the slider of the corresponding module, and its length can be changed by moving the slider; the variation range of the length is from $1.2 \mathrm{~m}$ to $1.5 \mathrm{~m}$. Six flexible rods are made of fiber glass, which not only guarantees the ability of bending and makes the manipulator lighter and thus safer, but also makes it have a certain strength to ensure its stable movement.

2.2. Analysis of the Prototype Machine. The biomimetic fish-fin continuum robot mechanism diagram is shown in

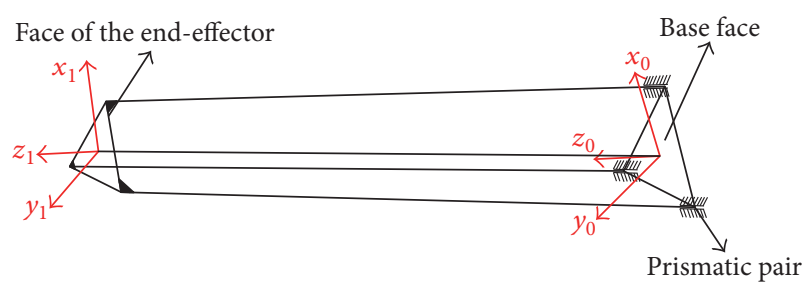

FIGURE 2: The mechanic diagram of the trunk of manipulator.

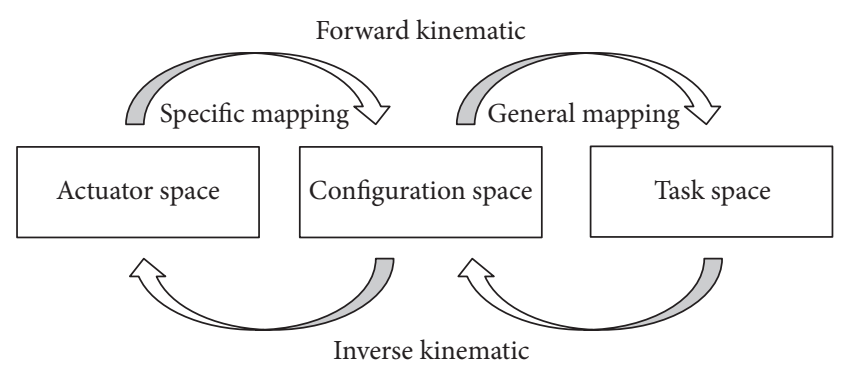

FIgURE 3: Three spaces and two mappings in the continuous kinematic model.

Figure 2, three black lines represent three active rods (6) of the prototype robot, the base face is fixed, three rods are connected with the end-effector, and the prismatic pair represents corresponding slider of the module. Due to the movement of the slider, it can change the length of each rod, respectively, which can also change the position and orientation of the end-effector. We establish the coordinate frames $x_{0}-y_{0}-z_{0}$ and $x_{1}-y_{1}-z_{1}$ on the base face (10) and face of end-effector (7), respectively.

As shown in Figure 2, we approximate the entire structure as a straight prism since the length of rod is much longer than the lengths of the sides of both faces, and we establish and analyze the kinematic model under this approximation.

\section{Constant Curvature Kinematic Model}

We used a kinematic model of continuous curvature based on geometry. Compared with the method of modified D-H and finite element analysis [14], we find that this method of modeling is more suitable for continuum robot and easier to implement. This kinematic model is based on two points:

(1) when the manipulator is bending, we can approximate as a circular arc;

(2) we ignore the gravity in the kinematic modeling.

The assumption of the continuous curvature kinematic model can be divided into two mappings, as visualized in Figure 3: a specific mapping is from actuation space to configuration space parameters and the other is from configuration space to task space.

3.1. The General Mapping between Configuration Space and Task Space. The mapping between configuration space and task space can be described by homogeneous transformation matrix from the base to the end-effector. We establish the 
corresponding coordinate frame as shown in Figure 1. At the same time, in Figure 4, we can see the kinematic model and coordinate frame of the manipulator.

We consider the $+z_{0}$-axis of the base coordinate frame to be tangent to the base of the robot, and the pedal is in the center of the base, the direction of the $z$-axis points to the top of the robot in the initial state, and the variable $\phi$ describes the angle of the rotation of the trunk about the $z_{0}$-axis. When $\phi=$ 0 , the direction of the robot trunk bending defines the $+x_{0^{-}}$axis, and $+y_{0}$-axis is defined by right-hand rule about $+x_{0^{-}}$axis and $+z_{0}$-axis.

We establish the terminal coordinate frame on center of the end-effector in the prototype robot; when the main body of robot rotates about $+z_{0}$-axis by $\phi$, the bending direction defines $+x_{1}$-axis. At the same time, we consider the $+z_{1}$-axis of the terminal coordinate frame to be tangent to the top of the main body of the robot. The direction of $+y_{1}$-axis is defined by right-hand rule about $+x_{1}$-axis and $+z_{1}$-axis.

In Figure 4, when $\phi=0$, the manipulator lies in the $x$ $z$ plane, its main body becomes a circular arc with radius of $r$ and its center at $\left(\begin{array}{lll}r & 0 & 0\end{array}\right)^{T}$, and then we can obtain the position of the end-effector as

$$
p=\left[\begin{array}{lll}
r(1-\cos \theta) & 0 & r \sin \theta
\end{array}\right]^{T}
$$

If we rotate the arc about $+z_{0}$-axis by angle of $\phi$, the homogenous transformation can be obtained as

$$
T=\left[\begin{array}{cc}
R_{z}(\phi) & 0 \\
0 & 1
\end{array}\right]\left[\begin{array}{cc}
R_{y}(\theta) & p \\
0 & 1
\end{array}\right]
$$

We note that, in some applications such as when a gripper is attached on the top of the robot, it is useful to orient the coordinate frame such that it aligns with the base frame when "sliding" along the arc to the base without rotation about the local $z$-axis, that is, use of Bishop's frame $[15,16]$. It is equivalent to postmultiplying $T$ by a homogeneous transformation with rotation $R_{z}(-\phi)$, and we can obtain the following equation:

$$
T_{w}=\left[\begin{array}{cccc}
\cos ^{2} \phi(\cos k s-1)+1 & -\sin \phi \cos \phi(\cos k s-1) & \cos \phi \sin k s & \frac{\cos \phi(1-\cos k s)}{k} \\
\sin \phi \cos \phi(\cos k s-1) & \cos ^{2} \phi(1-\cos k s)+\cos k s & \sin \phi \sin k s & \frac{\sin \phi(1-\cos k s)}{k} \\
-\cos \phi \sin k s & -\sin \phi \sin k s & \cos k s & \frac{\sin k s}{k} \\
0 & 0 & 0 & 1
\end{array}\right] .
$$

is

From (3), we obtain that the position of the end-effector

$$
\begin{aligned}
& p_{x}=\frac{\cos \phi(1-\cos \theta)}{k}, \\
& p_{y}=\frac{\sin \phi(1-\cos \theta)}{k} \\
& p_{z}=\frac{\sin \theta}{k}
\end{aligned}
$$

We can derive the inverse kinematic equation from (4) as follows:

$$
\begin{aligned}
& a=\frac{p_{y}^{2}}{p_{z}^{2} \sin ^{2} \phi}-1, \\
& \theta=\arccos \left(\frac{1-a}{a+1}\right), \\
& \phi=\arctan \left(\frac{p_{y}}{p_{x}}\right) .
\end{aligned}
$$

3.2. The Specific Mapping between Actuated Space and Configuration Space. The intention of deriving the mapping between actuation space and the configuration space is to obtain the relationship between the actuation states, $q=$ $\left(\begin{array}{lll}l_{1} & l_{2} & l_{3}\end{array}\right)$, and the parameters $\phi(q), k(q)$, and $l(q)$ of the constant curvature model, in which variable $\phi$ describes the rotation angle of the manipulator, variable $k$ describes the curvature, and variable $l$ describes the length of the arc. We can obtain the variable $\theta$ from $\theta=k l$.

From Figures 5 and 6 , we can derive the relationship between bending radius and the bending radius of each flexible rod in the following form:

$$
r_{i}=r-d \cos \phi_{i}
$$

The variable $d$ describes the distance from the center of the base face to the center of the flexible rod as shown in Figure 6. The variable $\phi_{i}$ describes the angle between bending direction of the manipulator and the location of the $i$ th rod. Recall that $l=\theta r$ and $l_{i}=\theta r_{i}$, and then we can obtain the relationship between the length of arc $(l)$ and the length of the $i$ th $\operatorname{rod}\left(l_{i}\right)$ as follows:

$$
l_{i}=l-\theta d \cos \phi_{i}
$$

We can obtain the relationship between $\phi_{i}$ and $\phi$ from Figure 6 (in degree):

$$
\phi_{i}=90+120(i-1)-\phi
$$

From (7), we have

$$
l(q)=\frac{l_{1}+l_{2}+l_{3}}{3}
$$




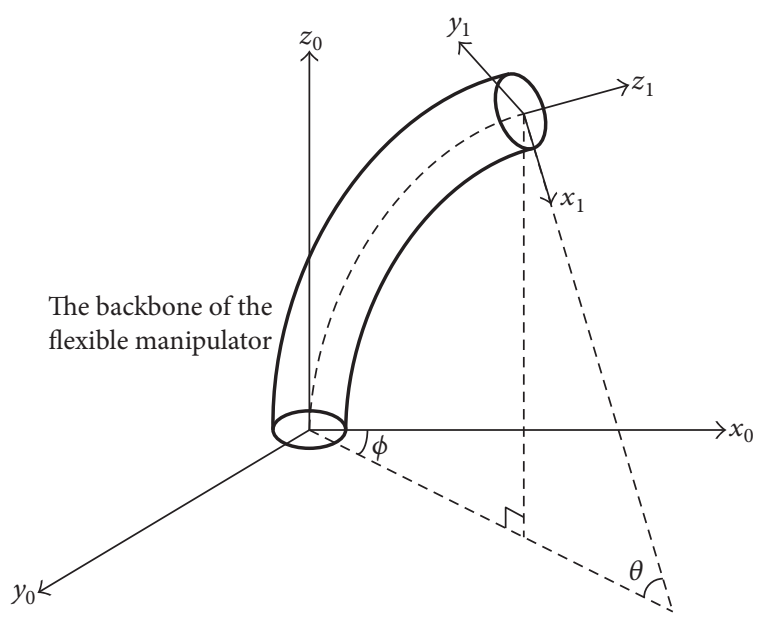

FIgURE 4: Kinematic model of the bionic robot.

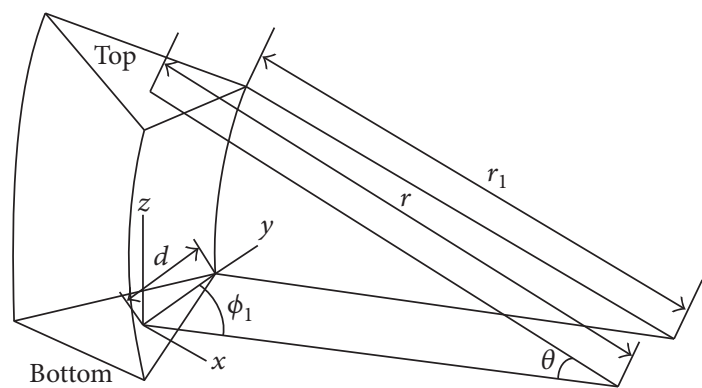

FIGURE 5: An illustration of the arc parameters is defined in the trunk.

Substituting $i=1,2$ and $i=2,3$ into (7), respectively, and combining them with (8), we obtain the relationship between $\phi$ and the actuation state $q$ :

$$
\phi(q)=\tan ^{-1}\left(\frac{\sqrt{3}\left(l_{2}+l_{3}-2 l_{1}\right)}{3\left(l_{2}-l_{3}\right)}\right) .
$$

Recall that $\theta=k l=l_{i} / r_{i}$, then we have $r_{i}=l_{i} / k l$, and simultaneous formulas with it and (6) give

$$
k=\frac{l-l_{i}}{l d \cos \phi_{i}} .
$$

Substituting $\phi_{1}=90-\phi$ into (9) gives

$$
k=\frac{l_{2}+l_{3}-2 l_{1}}{\left(l_{1}+l_{2}+l_{3}\right) d \sin \phi} .
$$

From (12) and (10), we can derive the relationship between the curvature $k$ and the actuated state $q$; that is,

$$
k(q)=\frac{2 \sqrt{l_{1}^{2}+l_{2}^{2}+l_{3}^{2}-l_{1} l_{2}-l_{1} l_{3}-l_{2} l_{3}}}{d\left(l_{1}+l_{2}+l_{3}\right)} .
$$

Equations (9), (10), and (13) describe the specific mapping of a single section of the continuum robot, and substituting

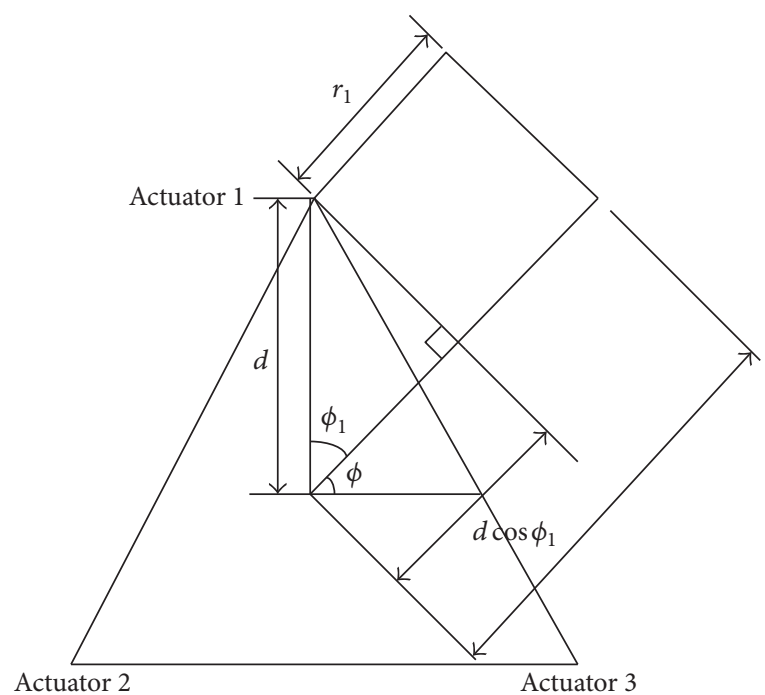

Figure 6: The diagram of the base section which is seen from top.

them into (4), we can obtain (15), which describes the relationship between the position of the end-effector and the length of three active rods.

$$
\begin{aligned}
& p_{x}=\frac{31.25 a(1-\cos [0.0106667 b])}{b \sqrt{1+c^{2} / 3 d^{2}}} \\
& p_{y}=\frac{18.04 c a(1-\cos [0.0106667 b])}{d b \sqrt{1+c / 3 d^{2}}}, \\
& p_{z}=\frac{18.04 c a}{d b \sqrt{1+c^{2} / 3 d^{2}}}
\end{aligned}
$$

In (14), $a, b, c$, and $d$ can be computed as follows:

$$
\begin{aligned}
& a=l_{1}+l_{2}+l_{3}, \\
& b=\sqrt{l_{1}^{2}-l_{1} l_{2}+l_{2}^{2}-l_{1} l_{3}-l_{2} l_{3}+l_{3}^{2}}, \\
& c=-2 l_{1}+l_{2}+l_{3}, \\
& d=l_{2}-l_{3} .
\end{aligned}
$$

\section{Velocity Kinematics}

There are different ways to compute the forward kinematics, as shown in Table 1, which represent the D-H parameters of the constant model, and (3) can be decomposed into several D-H transforms.

The relationship between actuate state $q$ and the endeffector state can be described as

$$
x=F_{(\mathrm{D}-\mathrm{H})}\left(F_{2}\left(F_{1}(q)\right)\right) \text {, }
$$

where $x$ is a vector which contains the information of the position and orientation about the end-effector. We can see that there are a set of functions including $F_{\mathrm{D}-\mathrm{H}}$, $F_{2}$, and $F_{1}$. These equations represent the modified D-H approach, the relationship between the $\mathrm{D}-\mathrm{H}$ parameters 
TABLE 1: D-H parameters for the continuum robot.

\begin{tabular}{lcclc}
\hline Link & $\theta$ & $D$ & $a$ & $\alpha$ \\
\hline 1 & $\phi$ & 0 & 0 & $-\pi / 2$ \\
2 & $k l / 2$ & 0 & 0 & $\pi / 2$ \\
3 & 0 & $d_{3}$ & 0 & $-\pi / 2$ \\
4 & $k l / 2$ & 0 & 0 & $\pi / 2$ \\
5 & $-\phi$ & 0 & 0 & - \\
\hline
\end{tabular}

$\left(\begin{array}{lllll}\theta_{1} & \theta_{2} & d_{3} & \theta_{4} & \theta_{5}\end{array}\right)^{T}$ and the curve parameters $\left(\begin{array}{lll}\phi & k & s\end{array}\right)^{T}$, and the relationship between three aforementioned parameters and the length of actuated flexible rods, which is represented with the vector of $q=\left(\begin{array}{lll}l_{1} & l_{2} & l_{3}\end{array}\right)^{T}$.

Then velocity kinematics are derived by differentiating (16) with respect to time. That is,

$$
\dot{x}=J(\dot{q})
$$

where $J$ is the Jacobian between the velocity of the endeffector and the time rate of length change.

From Table 1, we can use the general method to compute $J_{\mathrm{D}-\mathrm{H}}$. Then we can compute $J_{F_{2}}$ from Table 1 and $J_{F_{1}}$ from the function $F_{1}$.

Finally, the Jacobian $J$ can be computed by the following equation:

$$
J=J_{\mathrm{D}-\mathrm{H}} \cdot J_{F_{2}} \cdot J_{F_{1}} \cdot
$$

These detailed expressions of each term on the right side of (18) are shown in Appendix. Also, this approach of computing the Jacobian can be used for other continuum robots, such as the ones actuated pneumatically.

The Jacobian can help us with modeling of the dynamics of this kind of robot, and it is useful to control the robot with PID or other methods of implementation. These will be reported in future papers.

\section{The Analysis of the Manipulator Task Space}

The manipulator has three degrees of freedom, there are two types of special bending and one extension along $z$-axis, and we bend the manipulator by controlling the position of three slides to move the end-effector. We can simulate the task space of the manipulator through (4). We make the bending angle of the backbone change between 0 and $\pi / 2$ due to the material of fiber glass, and length of each active rod changes between 1.2 meters and 1.5 meters. According to this abovementioned rule, the task space of the manipulator is described as shown in Figure 7 by Matlab.

Figure 7 shows to us the positions which the end-effector can reach in the workspace.

\section{The Analysis of Kinematic Simulation}

6.1. The Simulation of Forward Kinematic. We can obtain the motion condition of each actuation rod. We define that the variable $d$ is $0.0625 \mathrm{~m}$, the bending angle is between $\pi / 6$ and

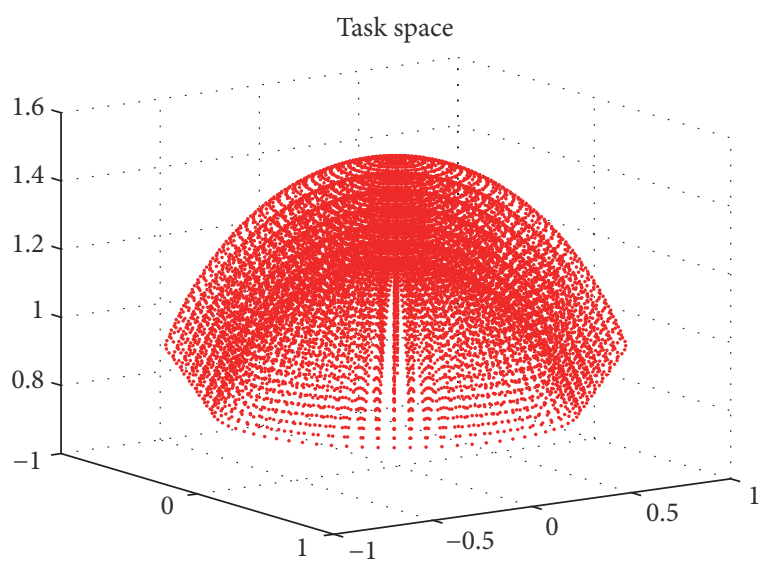

Figure 7: The simulation of the manipulator task space (unit: $\mathrm{m}$ ).

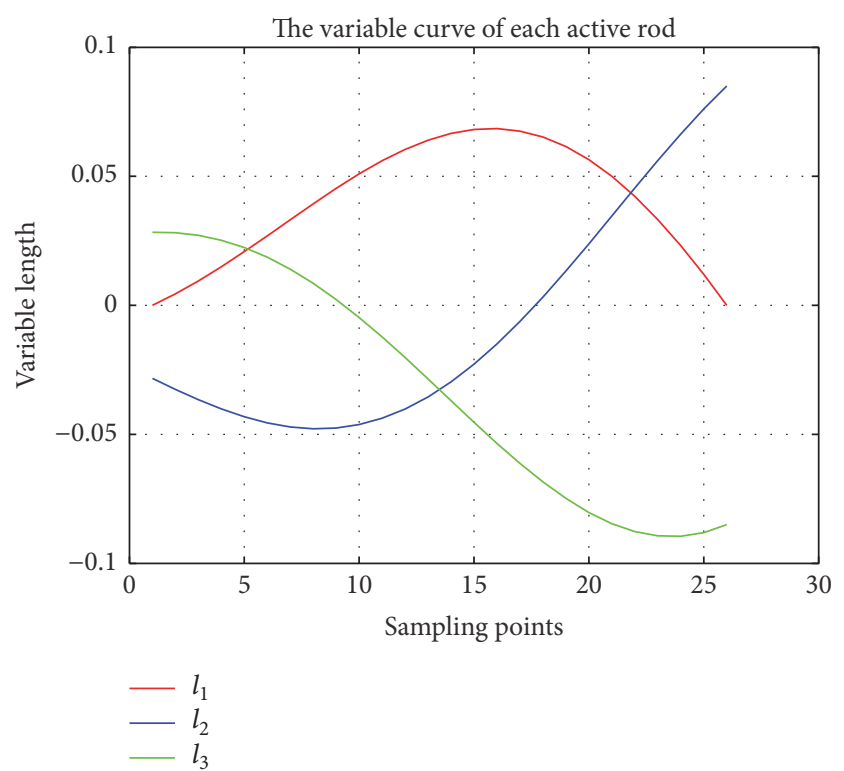

FIGURE 8: The simulation of the manipulator task space (unit: $\mathrm{m}$ ).

$\pi / 2$, and the angle $\phi$ is between 0 and $\pi$, when the number of sampling points is 25 . Using Matlab, the variable curve of each active rod is shown in Figure 8.

Under the same conditions, if we set that the initial positon of the end-effector is $(0,0,1.3)$ and when the length of the main body of the manipulator is 1.3 meters, we can simulate the position of end-effector in $x$-, $y$-, and $z$-axis as shown in Figure 9.

6.2. The Simulation of Inverse Kinematic. In this section, we assume a condition of the manipulator as the variable $\phi$ equals $\pi / 4$, the bending angle of the manipulator is between 0 and $\pi / 3$, such as $\pi / 36, \pi / 18, \pi / 12, \ldots, 5 \pi / 18,11 \pi / 36, \pi / 3$, and the length of the manipulator is 1.3 meters, then we compute the variable length of each active rod, $l_{1}, l_{2}, l_{3}$ correspondingly, and the simulation of inverse kinematic is shown as in Figure 10. 


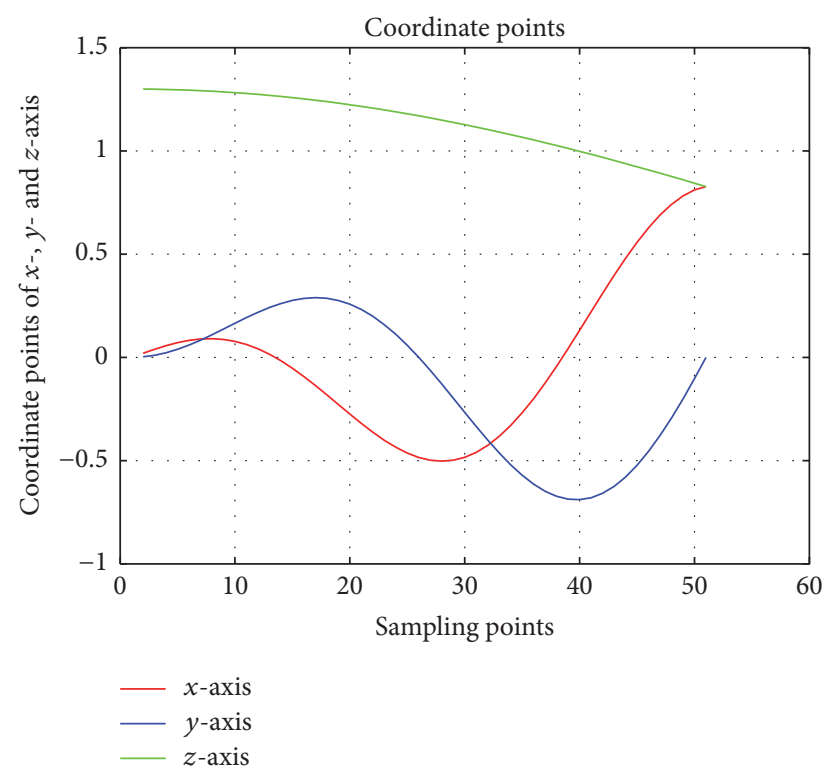

FIGURE 9: The variable curve of the position of the end-effector which is divided into $x$-, $y$-, and $z$-axis (unit: $\mathrm{m}$ ).

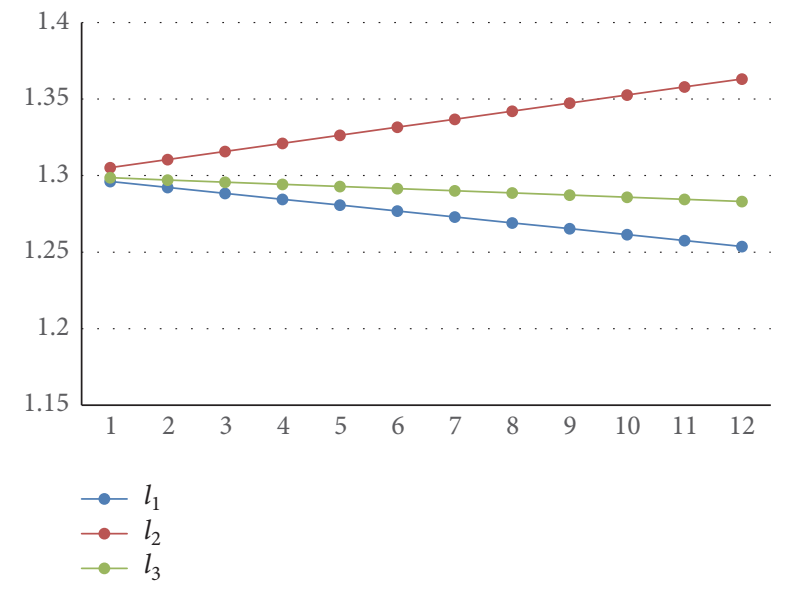

FIGURE 10: The simulation of the inverse kinematic (unit: $\mathrm{m}$ ).

We can derive the length of each active rod with knowing the position and orientation of the end-effector by analyzing the inverse kinematics, and it can help us to control the endeffector directly.

\section{Conclusion}

(1) Flexible continuum robots have been applied to a wide range of areas. Comparing them with traditional rigid robot, they are safer and more flexible. In this paper, we use constant curvature kinematic model based on geometry, and it is easy to implement and also suitable for robots with different kinds of actuation methods.

(2) We proposed a prototype robot based on the actuation of flexible rods, used the above-mentioned method to establish the kinematic model, and derive the inverse kinematic equations.

(3) In this paper, we analyze the kinematic model, derive the forward and inverse kinematic model, and the relationship between the length of each active rod and the position of the end-effector.

(4) The flexible continuum robot offers great potential for the robot application, and the manipulation is also an important issue. We will study the positioning error compensation method in future research to improve the accuracy.

\section{Appendix}

$J_{\mathrm{D}-\mathrm{H}}$ is

$$
J_{\mathrm{D}-\mathrm{H}}=\left[\begin{array}{ccccc}
-s_{1} s_{2} d_{3} & c_{1} c_{2} d_{3} & c_{1} s_{2} & 0 & 0 \\
c_{1} s_{2} d_{3} & s_{1} c_{2} d_{3} & s_{1} s_{2} & 0 & 0 \\
0 & -s_{2} d_{3} & c_{2} & 0 & 0 \\
0 & -s_{1} & 0 & -s_{1} & c_{1} s_{24} \\
0 & c & 0 & c_{1} & s_{1} s_{24} \\
1 & 0 & 0 & 0 & c_{24}
\end{array}\right]
$$

where $s_{i}$ means $\sin \theta_{i}, c_{i}$ means $\cos \theta_{i}, s_{i j}$ means $\sin \left(\theta_{i}+\theta_{j}\right)$, and $c_{i j}$ means $\cos \left(\theta_{i}+\theta_{j}\right)$.

Computing $J_{F_{2}}$ from Table 1 gives

$$
J_{F_{2}}=\left[\begin{array}{ccc}
1 & 0 & 0 \\
0 & \frac{s}{2} & \frac{k}{2} \\
0 & \frac{-2 \sin (k s / 2)+k s \cdot \cos (k s / 2)}{k^{2}} & \cos \frac{k s}{2} \\
0 & \frac{s}{2} & \frac{k}{2} \\
1 & 0 & 0
\end{array}\right] \text {. }
$$

Then we can compute the function $J_{F_{1}}$ from (9), (10), and (13) as follows:

$$
J_{F_{1}}=\left[\begin{array}{ccc}
-\frac{2 \sqrt{3}}{3 C \cdot\left(l_{2}-l_{3}\right)} & \frac{\left(\sqrt{3} / 3\left(l_{2}-l_{3}\right)\right)-D}{C} & \frac{\left(\sqrt{3} / 3\left(l_{2}-l_{3}\right)\right)+D}{C} \\
-A-\frac{l_{2}-2 l_{1}+l_{3}}{B} & -A-\frac{l_{2}-2 l_{1}+l_{3}}{B} & -A-\frac{l_{2}-2 l_{1}+l_{3}}{B} \\
\frac{1}{3} & \frac{1}{3} & \frac{1}{3}
\end{array}\right] \text {, }
$$


where $A, B, C, D$, and $E$ are given as

$$
\begin{aligned}
& A=\frac{2 E}{d\left(l_{1}+l_{2}+l_{3}\right)^{2}}, \\
& B=d\left(l_{1}+l_{2}+l_{3}\right) \cdot E, \\
& C=\frac{3\left(l_{2}-2 l_{1}+l_{3}\right)^{2}}{9\left(l_{2}-l_{3}\right)^{2}}+1, \\
& D=\frac{\sqrt{3}\left(l_{2}-2 l_{1}+l_{3}\right)}{3\left(l_{2}-l_{3}\right)^{2}}, \\
& E=\sqrt{l_{1}^{2}+l_{2}^{2}+l_{3}^{2}-l_{1} l_{2}-l_{1} l_{3}-l_{2} l_{3} .}
\end{aligned}
$$

Finally, we can compute the Jacobian $J$ by (18), Table 1 , and $g$ by (9), (10), and (13).

\section{Competing Interests}

The authors declare that they have no competing interests.

\section{Acknowledgments}

This research work is supported by National Key Technology Support Program of China (Grant no. 2015BAF10B01) and Science and Technology Commission of Shanghai Municipality (Grant nos. 15111104002 and 15111106302).

\section{References}

[1] S. Hirose and M. Mori, "Biologically inspired snake-like robots," in Proceedings of the IEEE International Conference on Robotics and Biomimetics (ROBIO '04), pp. 1-7, August 2004.

[2] M. W. Hannan and I. D. Walker, "Kinematics and the implementation of an elephant's trunk manipulator and other continuum style robots," Journal of Robotic Systems, vol. 20, no. 2, pp. 45-63, 2003.

[3] C. Laschi, B. Mazzolai, V. Mattoli, M. Cianchetti, and P. Dario, "Design of a biomimetic robotic octopus arm," Bioinspiration \& Biomimetics, vol. 4, no. 1, 2009.

[4] G. Robinson and J. B. C. Davies, "Continuum robots-a state of the art," in Proceedings of the IEEE International Conference on Robotics and Automation, pp. 2849-2854, Detroit, Mich, USA, May 1999.

[5] M. Csencsits, B. A. Jones, W. McMahan, V. Iyengar, and I. D. Walker, "User interfaces for continuum robot arms," in Proceedings of the IEEE/RSJ International Conference on Intelligent Robots and Systems (IROS '05), pp. 3123-3130, Edmonton, Canada, August 2005.

[6] $\mathrm{H}$. Hu, "Kinematic analysis and simulation for cable-driven continuum robot," Journal of Mechanical Engineering, vol. 46, no. 19, pp. 1-8, 2010.

[7] B. A. Jones and I. D. Walker, "Practical kinematics for realtime implementation of continuum robots," IEEE Transactions on Robotics, vol. 22, no. 6, pp. 1087-1099, 2006.

[8] I. D. Walker, C. Carreras, R. McDonnell, and G. Grimes, "Extension versus bending for continuum robots," International Journal of Advanced Robotic Systems, vol. 3, no. 2, pp. 171-178, 2006.
[9] B. A. Jones and I. D. Walker, "Kinematics for multisection continuum robots," IEEE Transactions on Robotics, vol. 22, no. 1, pp. 43-55, 2006.

[10] M. Rolf and J. J. Steil, "Constant curvature continuum kinematics as fast approximate model for the Bionic Handling Assistant," in Proceedings of the 25th IEEE/RSJ International Conference on Robotics and Intelligent Systems (IROS '12), pp. 3440-3446, October 2012.

[11] N. Simaan, "Snake-like units using flexible backbones and actuation redundancy for enhanced miniaturization," in Proceedings of the IEEE International Conference on Robotics and Automation, pp. 3012-3017, IEEE, Barcelona, Spain, April 2005.

[12] I. A. Gravagne, C. D. Rahn, and I. D. Walker, "Large deflection dynamics and control for planar continuum robots," IEEE/ASME Transactions on Mechatronics, vol. 8, no. 2, pp. 299307, 2003.

[13] N. Simaan, R. Taylor, and P. Flint, "A dexterous system for laryngeal surgery," in Proceedings of IEEE International Conference on Robotics and Automation (ICRA '04), vol. 1, pp. 351-357, New Orleans, La, USa, May 2004.

[14] B. A. Jones and I. D. Walker, "Limiting-case analysis of continuum trunk kinematics," in Proceedings of the IEEE International Conference on Robotics and Automation (ICRA '07), pp. 13631368, April 2007.

[15] R. J. Webster III and B. A. Jones, "Design and kinematic modeling of constant curvature continuum robots: a review," The International Journal of Robotics Research, vol. 29, no. 13, pp. 1661-1683, 2010.

[16] R. L. Bishop, "There is more than one way to frame a curve," The American Mathematical Monthly, vol. 82, pp. 246-251, 1975. 


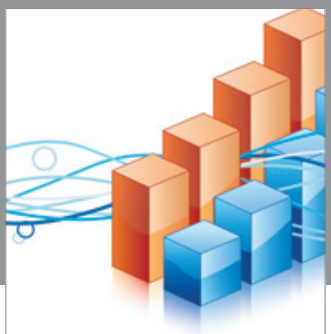

Advances in

Operations Research

vatem alat4

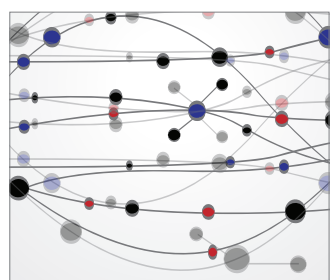

\section{The Scientific} World Journal
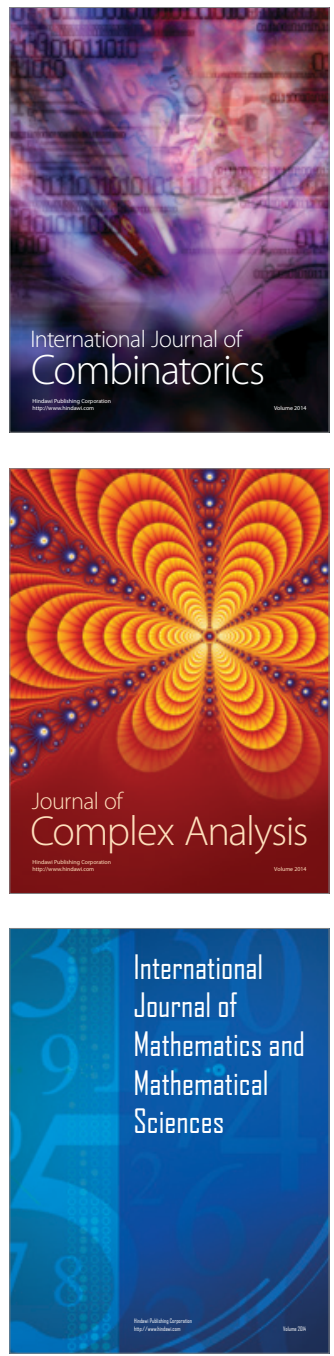
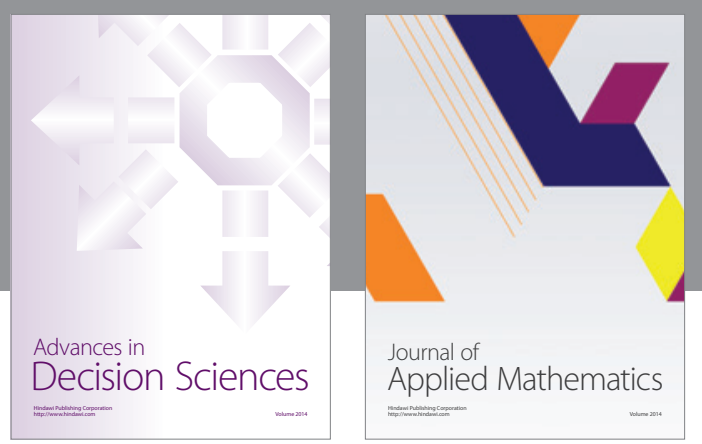

Algebra

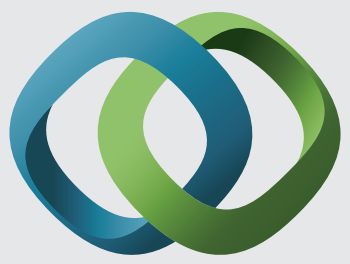

\section{Hindawi}

Submit your manuscripts at

http://www.hindawi.com
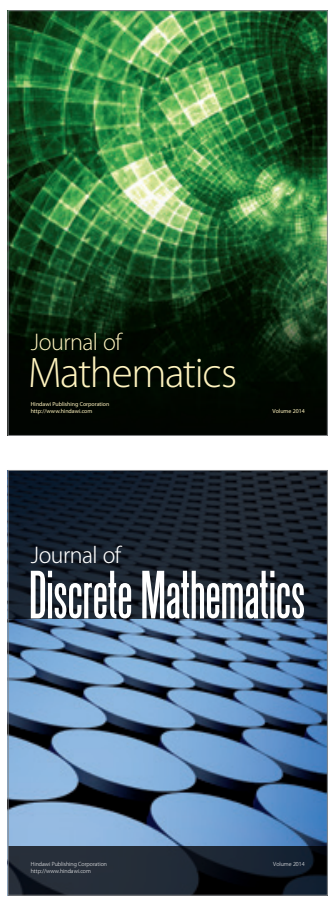

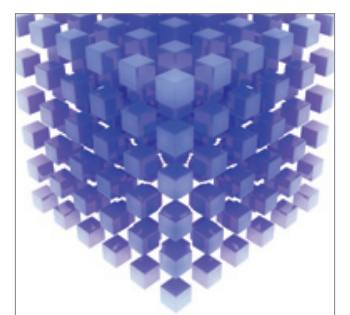

Mathematical Problems in Engineering
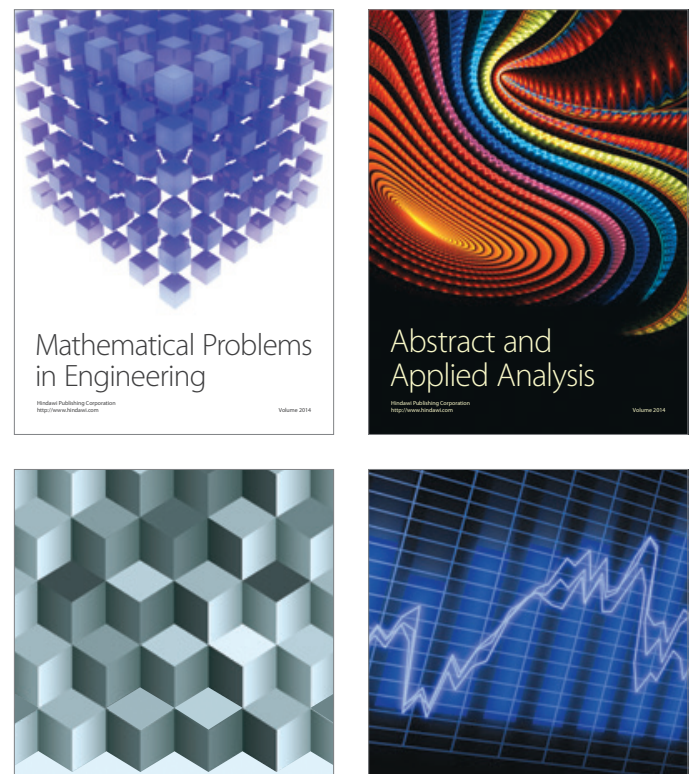

Journal of

Function Spaces

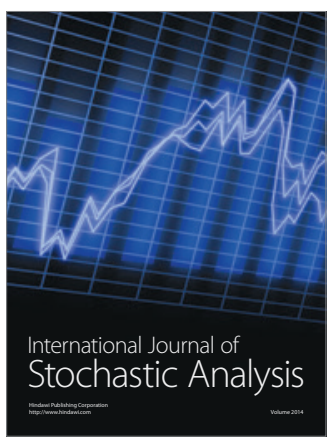

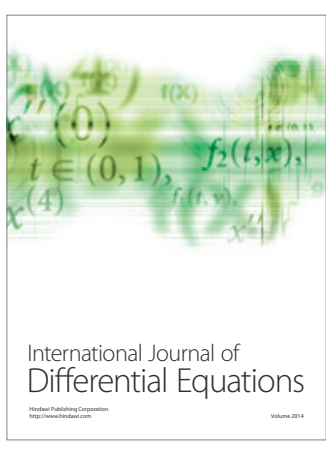
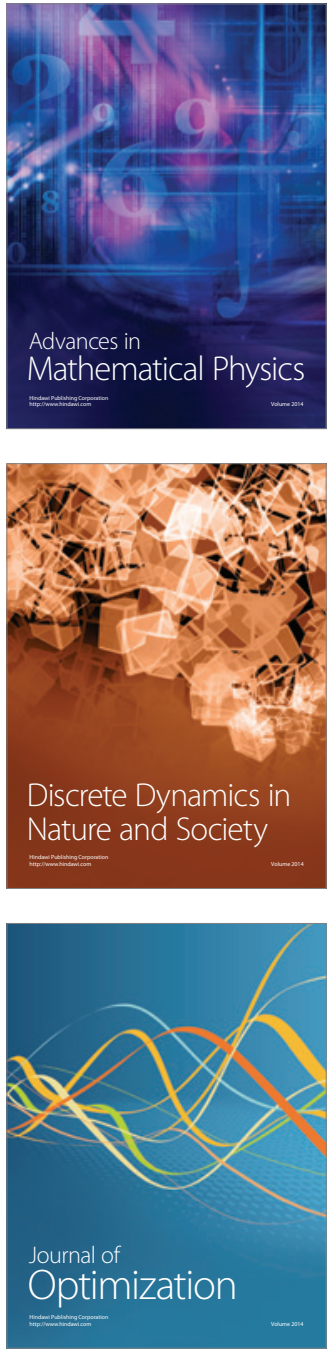